\title{
KNOWLEDGE OF AXILLARY BRACHIAL PLEXUS SONOANATOMY AMONGST ANAESTHETISTS
}

\author{
V. Woodham, K. Duraisamy, A. Pawa \\ Guy's and St Thomas' Hospital, London
}

\section{Background}

Ultrasound-guided regional anaesthesia is rapidly becoming standard practice, but its successful use is highly operator dependent.

Development of adequate skills is reliant upon various competencies, including correct image acquisition and interpretation.

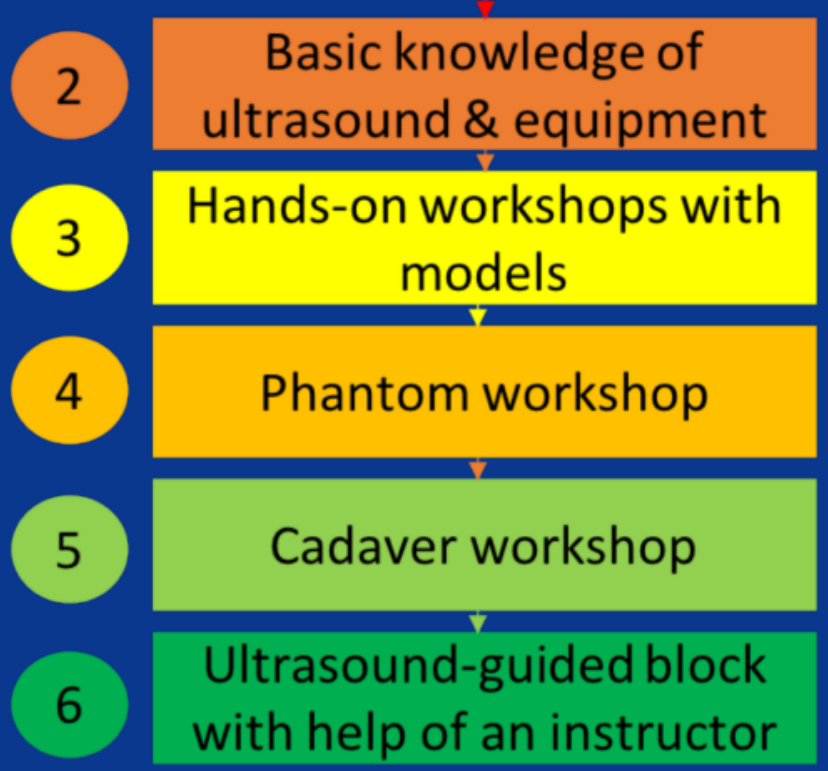

Fig. 1. Six-step teaching model for Regional Anaesthesia

The axillary brachial plexus block is considered a basic, low-difficulty block. As a superficial block with a low risk of complications, it is ideal for beginners; multiple needle adjustments maximise opportunity for needling practice.

Anaesthetists of all stages should therefore have thorough knowledge of sonoanatomy of the upper limb.

\section{Method}

20 anaesthetists of all grades were randomly selected to identify components of the axillary brachial plexus on a live model. They were given no more than 5 minutes scanning time and asked to identify nine key neurovascular and muscular anatomical structures.

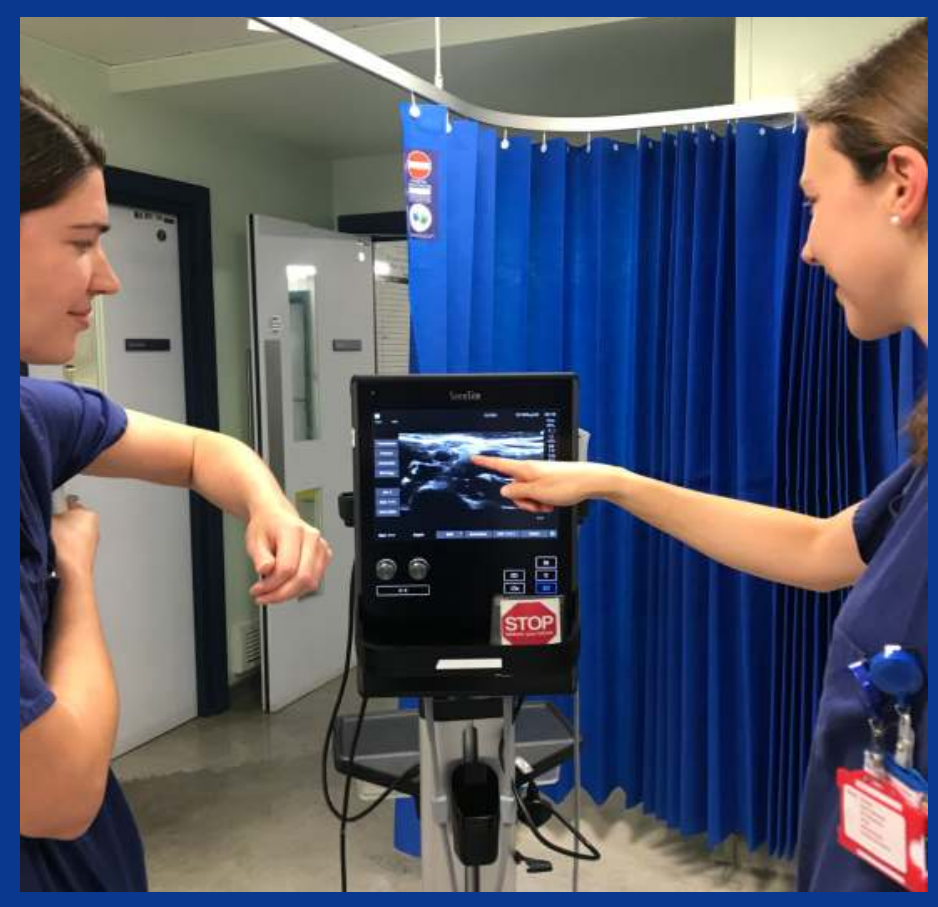

Fig. 2. Trainee anesthetist identifying structures in the axillary brachial plexus

\section{Results}

All participants were able to identify the axillary artery and vein. However, only $45 \%$ were able to successfully identify the median and radial nerves, with $\mathbf{4 0} \%$ correctly identifying the ulnar nerve. The musculocutaneous nerve was the most commonly identified structure $(\mathbf{7 5 \%})$, whilst the coracobrachialis muscle was the least (54\%). Speciality trainees (ST4-ST7) performed best, obtaining $78 \%$ of correct responses,

followed by Consultants $(60 \%)$. Core Trainees had the fewest correct responses (49\%).

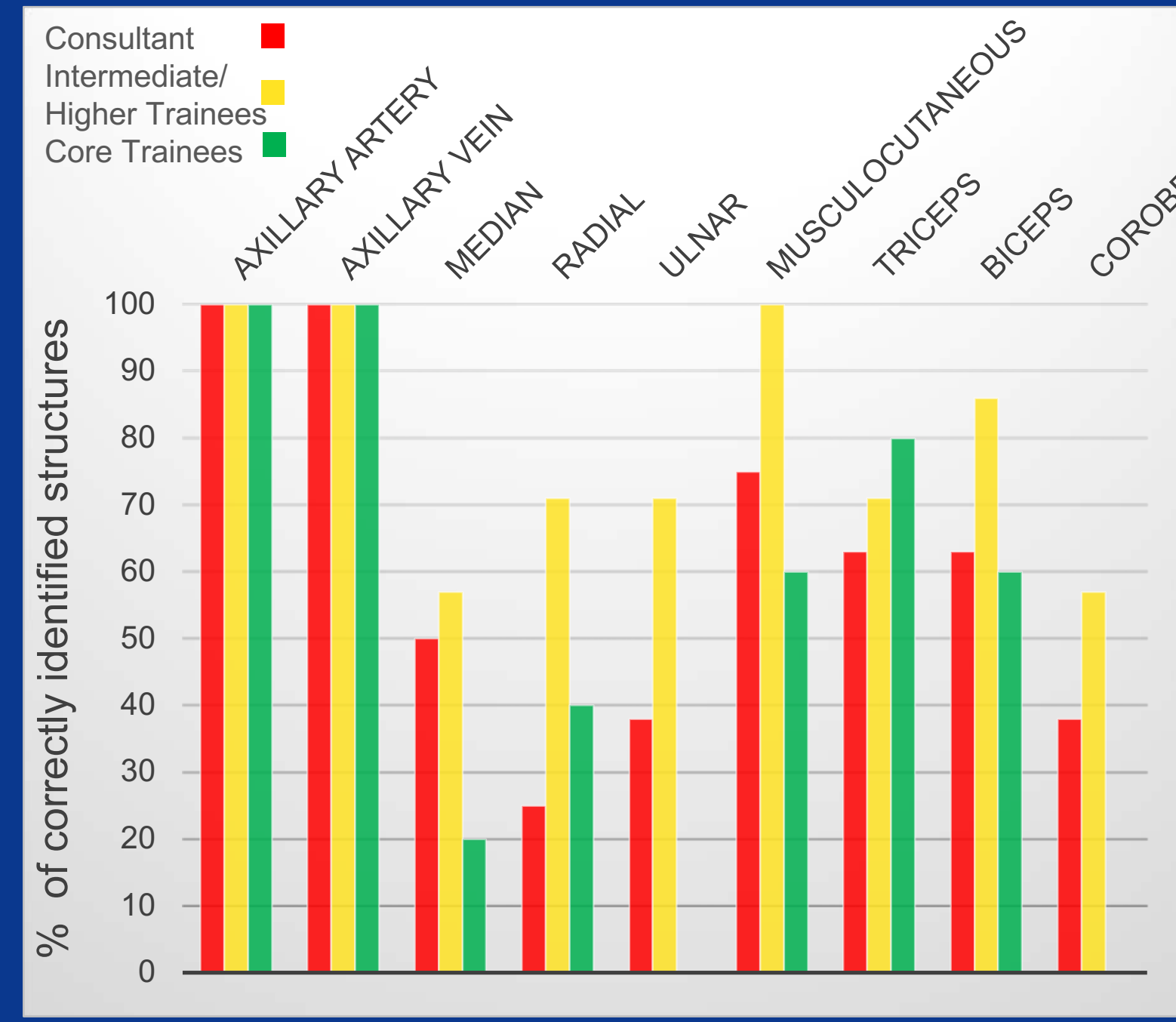

Fig. 3. Graph showing percentage of correctly identified structures in the axillary brachial plexus by different grades of anesthetist

\section{Discussion}

Despite the axillary brachial plexus block being simple and commonly performed, we identified a knowledge deficit around ultrasound anatomy across all grades.

This could lead to increased risk of patient harm with decreased success and reliability of blocks. This highlights the need for further education and increased frequency of formal teaching opportunities at all levels.

[1] Ultrasound-guided anaesthesia of peripheral nerves. The new challenge for anaesthesiologists. Kessler J, Marhofer, P, Rapp HJ, Hollmann MW. Anaesthetist 2007;56: 642-55

[2] Ultrasound in regional anaesthesia. Anaesthesia. J Griffin, B Nicholls

[3] The American Society of Regional Anesthesia and Pain Medicine and the European Society of Regional Anaesthesia and Pain Therapy Joint Committee Recommendations for Education and Training in Ultrasound-Guided Regional Anesthesia. B Sites, V Chan, J Neal, R Weller, T Grau.Regional Anaesesthesia and Pain Medicine. 